\section{SAT0517 FACTORS RELATED TO ANALGESIC USE IN PATIENTS WITH KNEE AND/OR HIP OSTEOARTHRITIS REFERRED TO AN OUTPATIENT CENTER: RESULTS FROM THE AMSTERDAM OSTEOARTHRITIS COHORT}

J. Knoop ${ }^{1}$, J. van Tunen ${ }^{2}$, M. van der Esch ${ }^{1}$, L.D. Roorda ${ }^{1}$, J. Dekker ${ }^{3}$, M. van der Leeden ${ }^{1,3}$, W.F. Lems ${ }^{4,5} .{ }^{1}$ Amsterdam Rehabilitation Research Center, Reade, Center for Rehabilitation and Rheumatology, Amsterdam, Netherlands; ${ }^{2}$ Department of Sports Science and Clinical Biomechanics, University of Southern Denmark, Odense, Denmark; ${ }^{3}$ Department of Rehabilitation Medicine, VU University Medical Center; ${ }^{4}$ Jan van Breemen Research Institute, Reade, Center for Rehabilitation and Rheumatology; ${ }^{5}$ Department of Rheumatology, VU University Medical Center, Amsterdam, Netherlands

Background: Analgesics are recommended in current guidelines, but non-use and inadequate prescription have been reported in patients with knee and/or hip osteoarthritis (OA). Furthermore, predictors of analgesic use have not yet been clarified.

Objectives: To (i) describe the use of analgesics; and (ii) determine factors that are related to analgesic use in patients with knee and/or hip OA referred to an outpatient center.

Methods: Data from 656 patients with knee and/or hip OA referred to an outpatient center (Amsterdam Osteoarthritis (AMS-OA) cohort) were used. Self-reported use of analgesic (yes/no) was administered and subdivided into acetaminophen, non-steroidal anti-inflammatory drugs (NSAIDs, including coxibs) and opioids. Logistic regression analyses were performed to analyze the association between analgesic use and disease-related, predisposing, and enabling factors.

Results: Analgesic use was reported by $62 \%$ of the patients, with acetaminophen, NSAIDs and opioid use reported by $50 \%, 30 \%$ and $12 \%$, respectively. Factors related to analgesic use were higher pain severity, longer duration of symptoms, higher radiographic hip OA severity, overweight/obesity, and psychological distress. These factors explained $21 \%$ of the variance of analgesic use.

Conclusions: Less than two-third of patients with knee and/or hip OA referred to an outpatient center reported analgesic use, which seems to be indicative for under-use. Although multiple, mostly disease-related factors were associated with analgesic use, it remained predominantly unexplained. Our findings may indicate that prescription of analgesics should be guided more dominantly by clinical symptoms and needs, and preceded by a thorough shared decision making process between patient and physician.

Disclosure of Interest: None declared

DOI: 10.1136/annrheumdis-2017-eular.2286

\section{SAT0518 ASSOCIATION BETWEEN GRIP STRENGTH AND HAND AND KNEE RADIOGRAPHIC OSTEOARTHRITIS IN OLDER ADULTS: DATA FROM THE DONG-GU STUDY}

J.-E. Kim, J.-H. Kang, K.-E. Lee, D.-J. Park, S.-S. Lee on behalf of Chonnam. Chonnam National University Medical School and Hospital, Gwangju, Korea, Republic Of

Objectives: Although some studies have shown a negative relationship between grip strength and hand osteoarthritis $(\mathrm{OA})$, little is known about how grip strength is related to specific radiographic features of hand $O A$, such as osteophytes, joint space narrowing, and erosion. In addition, no reported study has examined whether grip strength, as a measure of muscle activity, is related to knee OA, which may also show a one-way effect of reduced muscle strength on OA. In this large, population-based cohort study, we took advantage of the availability of subjects without hand pain to evaluate the effect of grip strength on OA using a novel, semi-quantitative grading system. We also examined whether grip strength was related to detailed radiographic features of $O A$.

Methods: Data from 2,251 subjects enrolled in the Dong-gu study, who had no hand joint pain, were analyzed to investigate the relationship between grip strength and OA. Hand grip strength was measured using a hand-held dynamometer, and radiographs of the hand and knee were scored according to a semi-quantitative grading system. Multiple linear regressions were used to explore associations between grip strength and radiographic features of $O A$.

Results: Grip strength in men and women was negatively related to hand (both $p<0.001$ ) and knee (men, $p<0.001$; women, $p=0.010$ ) OA after adjusting for confounders. Hand (men, $p<0.001$; women, $p=0.001$ ) and knee (both $p<0.001)$ joint space narrowing showed the strongest associations with low grip strength, regardless of sex. Moreover, the severity of hand osteophytes in women $(p=0.001)$, knee osteophytes in men $(p=0.006)$, hand malalignment (men, $p=0.008$; women, $p=0.041$ ), and subchondral cysts (men, $p<0.001$; women, $p$ $=0.007$ ) was correlated with low grip strength in both sexes.

Conclusions: Among subjects without hand joint pain, low grip strength was associated significantly with hand and knee radiographic OA, regardless of sex. Among all types of OA radiographic damage, low grip strength showed the strongest association with joint space narrowing.

Disclosure of Interest: None declared

DOI: 10.1136/annrheumdis-2017-eular.2355

\section{SAT0519 THE VALUE OF HIGH-SENSITIVITY C-REACTIVE PROTEIN IN HAND AND KNEE RADIOGRAPHIC OSTEOARTHRITIS: DATA FROM THE DONG-GU STUDY}

J.-E. Kim, J.-H. Kang, K.-E. Lee, D.-J. Park, S.-S. Lee ${ }^{1,1}$. Chonnam National University Medical School and Hospital, Gwangju, Korea, Republic Of

Objectives: Due to the inconsistent association between high-sensitivity Creactive protein (hs-CRP) and osteoarthritis $(\mathrm{OA})$, we evaluated the relationship between hs-CRP and various radiographic findings in older adults with $\mathrm{OA}$.

Methods: This cross-sectional study recruited 2,400 participants from the population-based Dong-gu cohort. The scores of radiographic features in OA on X-rays of the knees and hands were computed using a semi-quantitative grading system. The hs-CRP levels were measured using a particle-enhanced immunonephelometry assay. Correlations showing the relationship between hs-CRP and OA were calculated using multiple linear correlation analysis.

Results: The hs-CRP levels were significantly higher in older subjects ( $p$ $<0.001)$, those with a higher body mass index (BMI) $(p<0.001)$, current smokers $(p<0.001)$, current alcohol drinkers $(p=0.012)$, those who were less physically active $(p=0.002)$, and those with a lower level of education $(p=0.036)$. After adjusting for BMI and other confounders, the total OA scores (knee, $p=0.048$; hand, $p=0.010$ ), erosion scores (knee, $p=0.035$; hand, $p=0.031$ ), and sclerosis (knee, $p=0.021$; hand, $p=0.029$ ) in the knees and hands were all significantly positively correlated with hs-CRP. A significant association was also observed between hs-CRP and the hand malalignment score ( $p=0.012)$.

Conclusions: In this large cross-sectional study, the hs-CRP level was a significant predictor of radiographic OA. Of the various types of OA radiographic damage, erosion, sclerosis, and malalignment showed significant associations with hs-CRP.

Disclosure of Interest: None declared

DOI: 10.1136/annrheumdis-2017-eular.2358

\section{SAT0520 MARKED REDUCTION OF OSTEOARTHRITIS PAIN WITH A HYDROGEN SULFIDE-RELEASING NAPROXEN DERIVATIVE}

J.L. Wallace ${ }^{1}$, D. Vaughan ${ }^{2} .{ }^{1}$ University of Calgary, Calgary; ${ }^{2}$ Antibe

Therapeutics Inc, Toronto, Canada

Background: Nonsteroidal anti-inflammatory drugs (NSAIDs) remain a mainstay of therapy for osteoarthritis, but their use is limited by their propensity to cause significant gastrointestinal (GI) bleeding and ulceration. Hydrogen sulfide (H2S) is an endogenous signaling molecule that has been shown to exert protective and pro-healing effects in the GI tract. We developed a series of H2S-releasing NSAIDs, and have now tested the lead drug (ATB-346) in a phase 2a clinical trial in patients with osteoarthritis. ATB-346 is a derivative of naproxen, the most cardiovascular-safe of the NSAID family. Data from a phase 1 clinical trial suggested that ATB-346 was significantly more potent and long-lasting than naproxen in terms of suppressing cyclooxygenase (COX) activity.

Objectives: An open-label phase 2a trial was performed to determine if a low dose of ATB-346 (250 mg; equimolar to $160 \mathrm{mg}$ naproxen), given once daily, would provide significant pain relief in patients with osteoarthritis. To be admitted to the trial, patients had to exhibit $a \geq 10$ point increase in the WOMAC Visual Analog Score between their screening visit and the baseline study entry visit.

Methods: 12 patients with osteoarthritis of the knee who met the inclusion criteria were recruited for the trial (diagnosis of $\mathrm{OA} \geq 2$ years; age $40-75$; $\mathrm{BMI} \leq 40$ ). The WOMAC subscale pain score $(0-20)$ was the primary endpoint. The patients were off anti-inflammatory medication for 5 days prior to starting this study. After recording the initial WOMAC subscale pain score, they began once daily oral treatment with ATB-346 $(250 \mathrm{mg})$. Pain scores were recorded on the day prior to starting ATB-346 treatment ("day -1"), and on days 4 and 10 of treatment. Blood samples were collected for measurement of COX activity on days $-1,1,4$ and 10. Results: ATB-346 was safe and well tolerated. The mean WOMAC subscale pain score the day prior to initiation of treatment was $14.0+0.7$. A significant decrease in the WOMAC subscale pain score was observed on day $4(-4.3 \pm 1.0 ; p<0.01)$, with an even greater decrease on day $7(-7.6 \pm 1.5 ; \mathrm{p}<0.001)$. Whole blood COX activity was suppressed by more than $80 \%(p<0.01)$ on the first day of treatment, and continued to be suppressed throughout the study.

Conclusions: The results of this study demonstrate that a low dose of ATB-346, given only once daily, produces a clinically and statistically significant reduction of pain in patients with osteoarthritis, that may exceed what can be achieved with standard doses of napoxen or celecoxib $(1,2)$. The observed suppression of COX activity was consistent with the pain reduction data, supporting the conclusion that ATB-346 is considerably more potent and long-acting than naproxen. Extensive pre-clinical studies have demonstrated that ATB-346 has greatly improved GI safety as compared to naproxen and other commonly used NSAIDs.

References:

[1] Wittenberg et al. Arth Res Therap 2006; 8: R35 (doi: 10.1186/ar1854.

[2] Boucher M. http://www.page-meeting.org/pdf_assets/8269-Bayesian\%20Meta $\% 20$ Analysis\%20Final.pdf

Disclosure of Interest: J. Wallace Shareholder of: Antibe Therapeutics Inc, Employee of: Antibe Therapeutics Inc, D. Vaughan: None declared DOI: 10.1136/annrheumdis-2017-eular.1040 